\title{
Chitosan: A Desirable Candidate for Treating Hyperphosphatemia?
}

\author{
Haisong Zhang ${ }^{1,2, *}$, Meng $\mathrm{Yu}^{1}$ and Hailei Zhang ${ }^{2}$ \\ 1. Affiliated Hospital of Hebei University, Hebei University, Baoding, 071000, P. R. China \\ 2. College of Chemistry and Environmental Science, Hebei University, Baoding, 071002, P.R. China
}

\begin{abstract}
Non-absorbed macromolecular binders as sequestrants for phosphate ions offer an effective approach to treat hyperphosphatemia in ESRD (end-stage renal disease) patients. RenaGel ${ }^{\circledR}$ has been an example with remarkable success of a polymer synthesized to prevent the absorption of dietary phosphate for ESRD patients. Electrostatic interaction is the primary driving force for complexation of phosphate-based anions with these amino groups in the polymer backbone. Chitosan is a deacetylation product of chitin, which is the structural element in the exoskeleton of crustaceans and cell walls of fungi. The amino groups in the backbone give the phosphate binding ability to chitosan. This article has demonstrated that chitosan exhibited a phosphate binding effect indeed. Thus, it has potential applications in environmental management and wastewater treatment, as well as treatment of hyperphosphatemia patients.
\end{abstract}

Key words: Chitosan, phosphate binder, hyperphosphatemia.

\section{Introduction}

Recently, polymer pharmacy served as an inter-discipline subject involved with material science, polymer science, pharmacy, clinical medicine, analytical science and nanotechnology. It has seen significant growth since 1970s when the "polymer prodrug” concept was proposed by Ringsdorf [1]. In the initial stage, the applications of polymer pharmacy were limited to a few biological products such as interferon [2-4], heparin [5, 6] and serum albumin [7, 8]. With the development of pharmaceutics and nanotechnology, numerous novel kinds of polymers were synthesized or modified to meet the growing demands for pharmaceutical excipients. For examples, some polysaccharides especially modified celluloses were developed as sustained-release carriers in solid preparations aiming to achieve a smooth and long-acting release behavior $[9,10]$; Eudragit ${ }^{\circledR}$, the brand name for a kind of commercially available

Corresponding author: Haisong Zhang, Prof. Dr., No. 213 Yuhua Road, Department of Nephrology, Affiliated Hospital of Hebei University, Baoding, 071000, China, research field: biomacromolecule and nephrology. polymethacrylate-based biomaterials, has been widely used in preparing enteric preparations [11]; PLA (poly (lactic acid)) and PLGA (poly (lactic-co-glycolic acid)) approved by FDA (Food and Drug Administration) in 1980s have been used extensively for preparing sustained-release injectable microspheres or nanoparticles [12, 13]; some novel biopolymers were employed to achieve targeting preparations in nanoscale aiming to meet the "EPR effect" in tumor tissues $[14,15]$. Nowadays, the synthesized polymers are not merely used as excipients or framework material, but act as active pharmaceutical ingredients: RenaGel ${ }^{\circledR}$ (sevelamer hydrochloride), a hydrogel of cross-linked poly (allylamine hydrochloride), has been developed for the treatment of hyperphosphatemia [16, 17].

Hyperphosphatemia, an electrolyte disturbance in which there is an abnormal content increase of phosphate in the blood, is much common in patients with ESRD (end-stage renal disease) [18-20]. Generally, serum phosphorus levels in healthy adults can maintain a balance between intestine, bone, intracellular space and kidneys, and keep an average 
phosphorus level between $0.81 \mathrm{mmol} / \mathrm{L}$ and 1.45 $\mathrm{mmol} / \mathrm{L}$. But due to a serious renal insufficiency, the ESRD patients usually possess an abnormal mineral metabolism especially for serum phosphorus. A long-term abnormally elevated level of phosphate can produce secondary hyperparathyroidism, metabolism disorder of Vitamin D, renal osteopathy, cardiovascular complications and even an increased risk of death [21, 22]. The total dialytic phosphorus removal is only $800-1000 \mathrm{mg} /$ session or about 300 mg/day, suggesting an insufficient effect for patients with hyperphosphatemia [23]. So the treatment for patients with hyperphosphatemia has focused on using oral phosphate binders taken at mealtimes. Traditionally, calcium or aluminum-based agents were initially used as phosphate binders to reduce serum phosphate levels rely on the actions of ionic bonds. But the abnormally elevated level of aluminum caused by a long-term administration can result in increased neurological, hematologic and skeletal toxicity [24-27]; otherwise, the hypercalcemia may lead to serious cardiovascular diseases and soft-tissue calcification [28-30]. Later on, Fosrenol ${ }^{\circledR}$ (lanthanum carbonate) was developed to replace calcium-based preparations [31, 32]. But the side-effect of the increased lanthanum

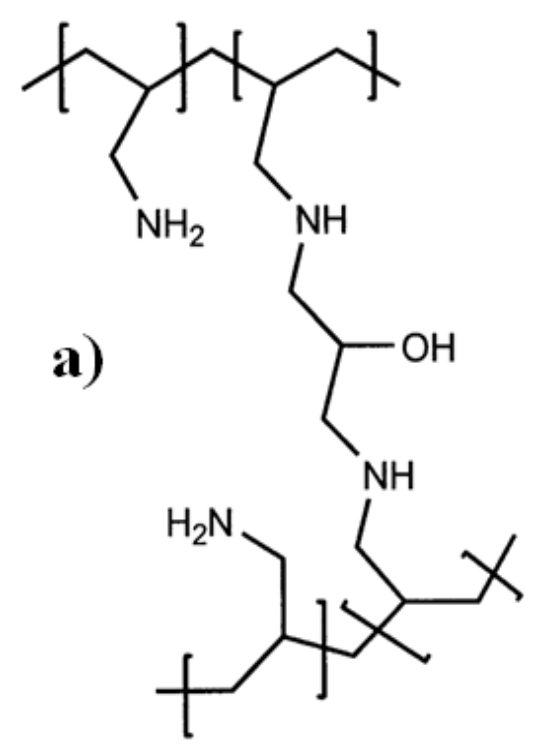

in blood is still not clear. Moreover, sometimes, medicinal charcoal tablets were also used as phosphate binders with low cost. But the lack of specificity limited its application in treatment of hyperphosphatemia [33, 34].

RenaGel ${ }^{\circledR}$, the first metal-free phosphate binder, is a hydrogel of cross-linked poly (allylamine hydrochloride) which is completely nonabsorbed and nondegradable from gastrointestinal tract. Since being approved in North America for a dozen years, RenaGel ${ }^{\circledR}$ has been an example with remarkable success of a polymer synthesized to prevent the absorption of dietary phosphate for ESRD patients. The binding capacity is based on the ionic hydrogen bond between phosphate anions and amines spaced on the polymer backbone (Fig. 1) [35]. But the key contraindications for sevelamer administration are a history of intestinal obstruction, followed by swallowing disorders [36]. So it is meaningful to search an amino-contained macromolecular binder with better biocompatibility. Chitosan, a linear polysaccharide composed of randomly distributed $\beta$-(1-4)-linked D-glucosamine and N-acetyl-D-glucosamine, may be a desirable candidate (Fig. 2).

b)

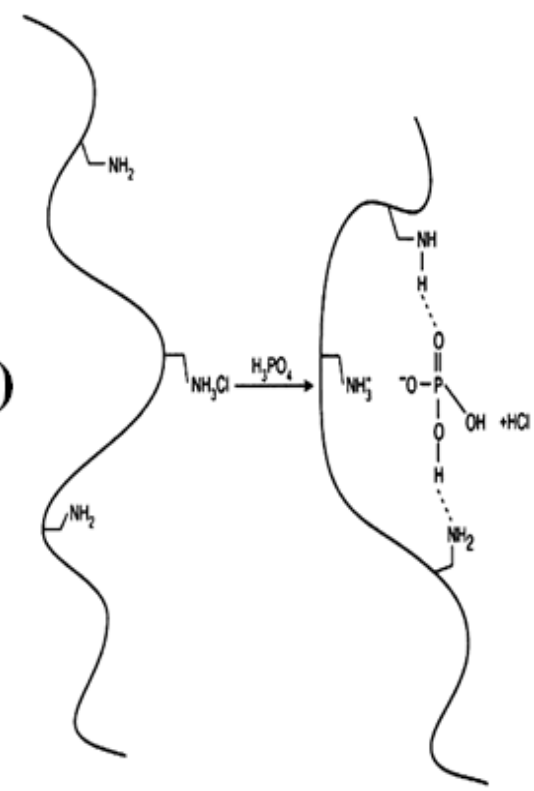

Fig. 1 a) Chemical structure of sevelamer; b) Binding interactions between polymeric amine gels and phosphate [35]. 


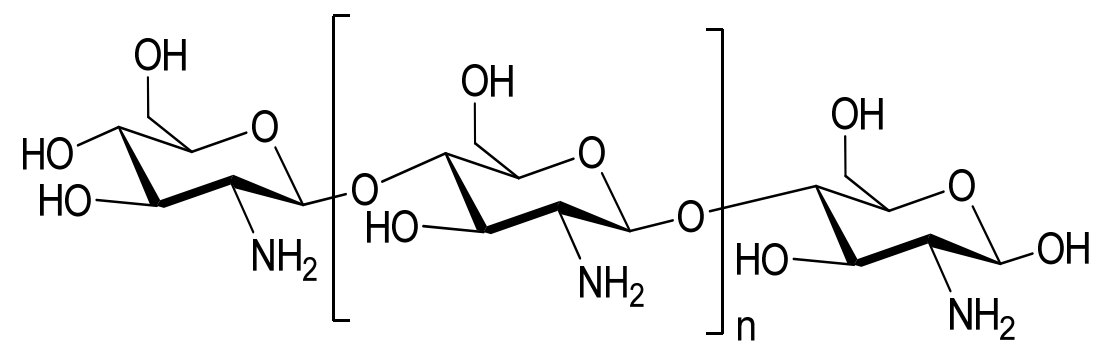

Fig. 2 Chemical structure of chitosan.

\section{Table 1 Reagent Composition.}

\begin{tabular}{ll}
\hline Contents & $\begin{array}{l}\text { Initial Concentration of } \\
\text { Solutions }\end{array}$ \\
\hline $\begin{array}{l}\text { R1a. Blacnnk Reagent } \\
\text { Sulphuric acid }\end{array}$ & $0.36 \mathrm{~mol} / \mathrm{L}$ \\
$\begin{array}{l}\text { Sodium chloride } \\
\text { Detergent }\end{array}$ & $154 \mathrm{mmol} / \mathrm{L}$ \\
R1b. Molybdate Reagent & $\mathrm{N} / \mathrm{A}$ \\
Ammonium molybdate & $3.5 \mathrm{mmol} / \mathrm{L}$ \\
Sulphuric acid & $0.36 \mathrm{mmol} / \mathrm{L}$ \\
Sodium chloride & $154 \mathrm{mmol} / \mathrm{L}$ \\
\hline
\end{tabular}

\section{Experimental Section}

\subsection{Materials}

Chitosan was purchased from Shanghai Makclin Biochemical Co., Ltd. RenaGel@ (sevelamer hydrochloride tablets) were obtained from Sanofi-Aventis group.

\subsection{Phosphate Binding Capability Studies}

The in vitro phosphate binding studies were performed in $200 \mathrm{~mL}$ of $\mathrm{KH}_{2} \mathrm{PO}_{4} / \mathrm{NaOH}$ buffer solution (1.6 mmol/L for phosphate, $\mathrm{pH}=6.8)$ thermostatically maintained at $37 \pm 0.5{ }^{\circ} \mathrm{C}$ based on Chinese Pharmacopoeia (2015 Ed.) Method III. Paddle rotational speed was set to $100 \mathrm{rpm}$. The phosphate concentrations were chosen to be equal to plasma phosphate level in a healthy human which is 1.6 $\mathrm{mmol} / \mathrm{L}$. The sample containing $200 \mathrm{mg}$ active ingredient was added to the dissolution cup. $5 \mathrm{~mL}$ samples were withdrawn at predetermined time intervals in order to understand the rate of ion binding onto $-\mathrm{NH}_{2}$ in vitro. The sample solutions were centrifuged and analyzed using an automatic biochemistry analyzer (ARCHITECT C8000).

\subsection{Analytical Methods}

An automatic biochemistry analyzer (ARCHITECT C8000) was employed to determine the phosphate levels of the liquid samples. Inorganic phosphprous detection kits (RANDOX ${ }^{\circledR}$ ) were used in this study; in addition, details of the reagent composition were shown in Table 1. The phosphate concentration of standard solution was $1.65 \mathrm{mmol} / \mathrm{L}$. Before test, the sample solution and standard solution were diluted with 100 times (v/v) amount of working reagent. The working reagent was prepared by mixing R1a and R1b in a feed ratio of 7:3 (v/v). The absorbance was determined at a wavelength of $340 \mathrm{~nm}$. The phosphate concentration of liquid sample was calculated according to the Lambert-Beer theory.

\section{Results and Discussion}

Generally, the phosphate binding behaviors mainly take place in intestines in vivo. So the in vitro phosphate binding studies were measured by immersing the ground chitosan power into a $\mathrm{KH}_{2} \mathrm{PO}_{4} / \mathrm{NaOH}$ buffer solution $(1.6 \mathrm{mmol} / \mathrm{L}$ for phosphate, $\mathrm{pH}=6.8$ ) of which the $\mathrm{pH}$ value is close to that of artificial intestinal juice. A dissolution tester equipped with six paddles was employed to simulate the gastrointestinal motilities. The BA (binding amount) of chitosan to phosphate was calculated according to the equation below:

$$
\mathrm{BA}=\left(\mathrm{C}_{0}-\mathrm{C}_{\mathrm{t}}\right) \mathrm{VM} \text { (phosphate) } / \mathrm{W}
$$

where MP is the molecular weight of the phosphate ion, $V$ is the volume of $\mathrm{KH}_{2} \mathrm{PO}_{4} / \mathrm{NaOH}$ buffer solution and $W$ is the weight of sample. When $C_{\mathrm{t}}$ became a constant, 


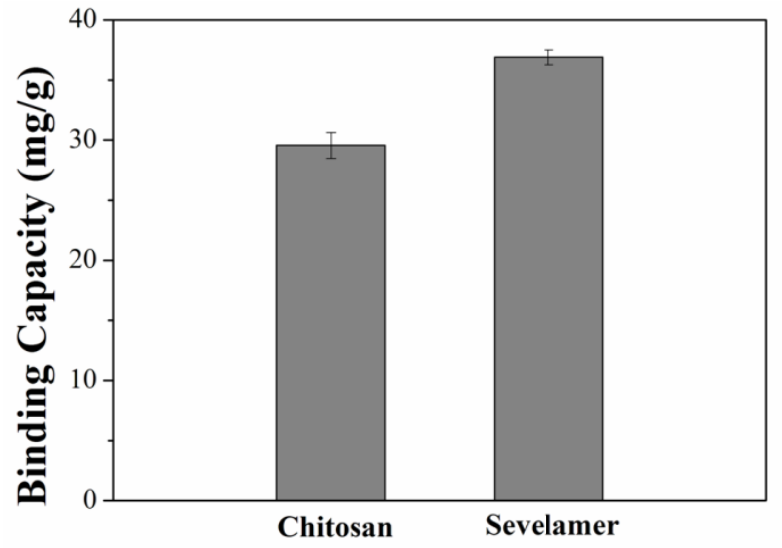

Fig. 3 Phosphate binding capacity of chitosan and Sevelamer.

the binding amount became the binding capacity.

Fig. 3 shows the phosphate binding capacity of chitosan; in addition, the phosphate binding capability of Sevelamer was also measured under the same conditions as reference. The results clearly show that the chitosan exhibited a phosphate binding effect indeed. Despite the binding capacity of chitosan cannot compare with that of Sevelamer, the much lower cost makes the natural organic material-based chitosan possess huge market potential in phosphate binding materials.

However, it's worth noting that there is a certain amount of lysozyme in a man's body. Chitosan can be degraded into oligosaccharides and even monosaccharides under lysozyme catalyzing. So chitosan cannot serve as a nonabsorbed binder as same as Sevelamer. When chitosan is degraded into oligosaccharides or monosaccharides, the binded phosphorus may also be absorbed in gastrointestinal tract followed by burst into systemic circulation. The absorbed phosphorus-containing saccharides can lead to an increase in serum phosphate directly. For ESRD patients, the administration of chitosan may be useless for treating hyperphosphatemia if the chitosan possess a high degradation rate in gastrointestinal tract. But it is hard to measure the degradation rate of chitosan under lysozyme catalyzing. Therefore, an in-vivo phosphate binding effect needs to be investigated. A detailed pharmacodynamic test will be conducted on sprague dawley with renal failure model to measure whether the chitosan can serve as an oral phosphate binder. Despite chitosan might not be a desirable phosphate binder for treating hyperphosphatemia, it can also have potential applications in environmental management and wastewater treatment.

\section{Conclusions}

In this study, we have confirmed that chitosan indeed possess a phosphate binding effect in vitro. The in-vivo binding effect of chitosan towards phosphate will be conducted to verify whether it has a therapeutic effect for hyperphosphatemia. If so, chitosan can serve as a desirable oral phosphate binding agent for treating hyperphosphatemia in ESRD patients due to its cheapness and good biocompatibility.

\section{Reference}

[1] Gros, L., Ringsdorf, H., and Schupp, H. 1981. "Polymeric Antitumor Agents on a Molecular and on a Cellular Level.” Angew. Chem. Int. Edit. 20: 305-25.

[2] Gray, P. W., Leung, D. W., Pennica, D., Yelverton, E., Najarian, R. Simonsen, C. C., Derynck, R., Sherwood, P. J., Wallace, D. M., Berger, S. L., et al. 1982. “Expression of Human Immune Interferon Cdna in E. Coli and Monkey Cells.” Nature 295: 503-8.

[3] Aggarwal, B. B., Eessalu, T. E., and Hass, P. E. 1985. "Characterization of Receptors for Human Tumour Necrosis Factor and Their Regulation by Gamma-interferon.” Nature 318: 665-7.

[4] Meda, L., Cassatella, M. A., Szendrei, G. I., Otvos, L., Jr., Baron, P., Villalba, M., Ferrari, D., and Rossi, F. 1995. "Activation of Microglial Cells by Beta-amyloid Protein and Interferon-Gamma.” Nature 374: 647-50.

[5] Petitou, M., Lormeau, J. C., and Choay, J. 1991. "Chemical Synthesis of Glycosaminoglycans: New Approaches to Antithrombotic Drugs.” Nature 350: 30-3.

[6] Aviezer, D., Hecht, D., Safran, M., Eisinger, M., David, G., and Yayon, A. 1994. "Perlecan, Basal Lamina Proteoglycan, Promotes Basic Fibroblast Growth Factor-Receptor Binding, Mitogenesis, and Angiogenesis.” Cell 79: 1005-13.

[7] Halliwell, B. 1988. "Albumin-An Important Extracellular Antioxidant?” Biochemical Pharmacology 37: 569-71.

[8] Kratz, F. 2008. "Albumin as a Drug Carrier: Design of Prodrugs, Drug Conjugates and Nanoparticles.” Journal of Controlled Release 132: 171-83. 
[9] Siepmann, J., and Peppas, N. A. 2012. "Modeling of Drug Release from Delivery Systems Based on Hydroxypropyl Methylcellulose (Hpmc).” Advanced Drug Delivery Reviews 64: 163-74.

[10] Siepmann, F., Siepmann, J., Walther, M., MacRae, R. J., and Bodmeier, R. 2008. "Polymer Blends for Controlled Release Coatings.” Journal of Controlled Release 125: 1-15.

[11] Thakral, S., Thakral, N. K., and Majumdar, D. K. 2013. “Eudragit (r): A Technology Evaluation.” Expert Opinion on Drug Delivery 10: 131-49.

[12] Jain, R. A. 2000. "The Manufacturing Techniques of Various Drug Loaded Biodegradable Poly (lactide-co-glycolide) (plga) Devices.” Biomaterials 21: 2475-90.

[13] Vasir, J. K., and Labhasetwar, V. 2007. "Biodegradable Nanoparticles for Cytosolic Delivery of Therapeutics.” Advanced Drug Delivery Reviews 59: 718-28.

[14] Matsumura, Y. 2008. "Poly (Amino acid) Micelle Nanocarriers in Preclinical and Clinical Studies." Advanced Drug Delivery Reviews 60: 899-914.

[15] Lukyanov, A. N., and Torchilin, V. P. 2004. "Micelles from Lipid Derivatives of Water-Soluble Polymers as Delivery Systems for Poorly Soluble Drugs.” Advanced Drug Delivery Reviews 56: 1273-89.

[16] Chertow, G. M., Burke, S. K., Lazarus, J. M., Stenzel, K. H., Wombolt, D., Goldberg, D., Bonventre, J. V., and Slatopolsky, E. 1997. "Poly Allylamine Hydrochloride (Renagel): A Noncalcemic Phosphate Binder for the Treatment of Hyperphosphatemia in Chronic Renal Failure.” Am. J. Kidney Dis. 29: 66-71.

[17] Slatopolsky, E. A., Burke, S. K., and Dillon, M. A. 1999. "Renagel, a Nonabsorbed Calcium- and Aluminum-Free Phosphate Binder, Lowers Serum Phosphorus and Parathyroid Hormone. The Renagel Study Group.” Kidney Int. 55: 299-307.

[18] Block, G. A., and Port, F. K. 2000. "Re-evaluation of Risks Associated with Hyperphosphatemia and Hyperparathyroidism in Dialysis Patients: Recommendations for a Change in Management.” Am. J. Kidney Dis. 35: 1226-37.

[19] Locatelli, F., Del Vecchio, L., Violo, L., and Pontoriero, G. 2014. "Phosphate Binders for the Treatment of Hyperphosphatemia in Chronic Kidney Disease Patients on Dialysis: A Comparison of Safety Profiles.” Expert Opin. Drug Saf. 13: 551-61.

[20] Rennick, A., Kalakeche, R., Seel, L., and Shepler, B. 2013. "Nicotinic Acid and Nicotinamide: A Review of Their Use for Hyperphosphatemia in Dialysis Patients." Pharmacotherapy 33: 683-90.

[21] Block, G. A., Hulbert-Shearon, T. E., Levin, N. W., and Port, F. K. 1998. "Association of Serum Phosphorus and
Calcium x Phosphate Product with Mortality Risk in Chronic Hemodialysis Patients: A National Study.” Am. J. Kidney Dis. 31: 607-17.

[22] Block, G. A., Klassen, P. S., Lazarus, J. M., Ofsthun, N., Lowrie, E. G., and Chertow, G. M. 2004. "Mineral Metabolism, Mortality, and Morbidity in Maintenance Hemodialysis.” J. Am. Soc. Nephrol. 15: 2208-18.

[23] Uribarri, J. 2007. "Phosphorus Homeostasis in Normal Health and in Chronic Kidney Disease Patients with Special Emphasis on Dietary Phosphorus Intake.” Semin. Dialysis 20: 295-301.

[24] Berlyne, G. M., Yagil, R., Ari, J. B., Weinberger, G., Knopf, E., and Danovitch, G. M. 1972. “Aluminium Toxicity in Rats.” Lancet 1: 564-8.

[25] Elliott, H. L., Dryburgh, F., Fell, G. S., Sabet, S., and Macdougall, A. I. 1978. "Aluminium Toxicity during Regular Haemodialysis.” Brit. Med. J. 1: 1101-3.

[26] Alfrey, A. C., Hegg, A., and Craswell, P. 1980. "Metabolism and Toxicity of Aluminum in Renal Failure." Am. J. Clin. Nutr. 33: 1509-16.

[27] Wang, M., Hercz, G., Sherrard, D. J., Maloney, N. A., Segre, G. V., and Pei, Y. 1995. "Relationship between Intact 1-84 Parathyroid Hormone and Bone Histomorphometric Parameters in Dialysis Patients without Aluminum Toxicity.” Am. J. Kidney Dis. 26: 836-44.

[28] Shigematsu, T., Negi, S., and Grp, C. R. 2012. “Combined Therapy with Lanthanum Carbonate and Calcium Carbonate for Hyperphosphatemia Decreases Serum FGF-23 Level Independently of Calcium and pth (Colc Study).” Nephrol. Dial. Transplant. 27: 1050-4.

[29] Qunibi, W. Y., Hootkins, R. E., McDowell, L. L., Meyer, M. S., Simon, M., Garza, R. O., Pelham, R. W., Cleveland, M. V. B., Muenz, L. R., He, D. Y., et al. 2004. “Treatment of Hyperphosphatemia in Hemodialysis Patients: The Calcium Acetate Renagel Evaluation (Care Study).” Kidney Int. 65: 1914-26.

[30] Nolan, C. R., and Qunibi, W. Y. 2003. "Calcium Salts in the Treatment of Hyperphosphatemia in Hemodialysis Patients.” Curr. Opin. Nephrol. Hy. 12: 373-9.

[31] Joy, M. S., Finn, W. F., and Grp, L. A. M. S. 2003. "Randomized, Double-Blind, Placebo-Controlled, Dose-Titration, Phase iii Study Assessing the Efficacy and Tolerability of Lanthanum Carbonate: A New Phosphate Binder for the Treatment of Hyperphosphatemia." American Journal of Kidney Diseases 42: 96-107.

[32] Huang, W., Liu, J., Tang, Y., Gao, X., Di, B., and Zhang, F. 2014. "Efficacy and Tolerability of Lanthanum Carbonate in Treatment of Hyperphosphatemia Patients Receiving Dialysis-A Systematic Review and Meta-analysis of Randomized Controlled Trials.” Current Medical Research and Opinion 30: 99-108. 
[33] Rutten, J., van den Berg, B., van Gelder, T., and van Saase, J. 2005. "Severe Theophylline Intoxication: A Delay in Charcoal Haemoperfusion Solved by Oral Activated Charcoal.” Nephrol. Dial. Transplant. 20: 2868-9.

[34] Christophersen, A. B., Levin, D., Hoegberg, L. C., Angelo, H. R., and Kampmann, J. P. 2002. "Activated Charcoal Alone or after Gastric Lavage: A Simulated Large Paracetamol Intoxication.” Brit. J. Clin. Pharmaco. 53: 312-7.
[35] Dhal, P. K., Holmes-Farley, S. R., Huval, C. C., and Jozefiak, T. H. 2006. "Polymers as Drugs.” Adv. Polym. Sci. 192: 9-58.

[36] Swanson, B. J., Limketkai, B. N., Liu, T. C., Montgomery, E., Nazari, K., Park, J. Y., Santangelo, W. C., Torbenson, M. S., Voltaggio, L., Yearsley, M. M., et al. 2013. "Sevelamer Crystals in the Gastrointestinal Tract (Git) a New Entity Associated with Mucosal Injury.” Am. J. Surg. Pathol. 37: 1686-93. 